\title{
Multiport Technology: The New Rise of an Old Concept
}

\author{
Serioja Ovidiu Tatu, Adriana Serban, Mohamed Helaoui and Alexander Koelpin
}

\section{Linköping University Post Print}

\section{Tweet}

N.B.: When citing this work, cite the original article.

Serioja Ovidiu Tatu, Adriana Serban, Mohamed Helaoui and Alexander Koelpin, Multiport Technology: The New Rise of an Old Concept, 2014, IEEE Microwave Magazine, (15), 7, 3444.

\section{http://dx.doi.org/10.1109/MMM.2014.2356149}

(C)2014 IEEE. Personal use of this material is permitted. However, permission to reprint/republish this material for advertising or promotional purposes or for creating new collective works for resale or redistribution to servers or lists, or to reuse any copyrighted component of this work in other works must be obtained from the IEEE.

\section{http://ieeexplore.ieee.org/}

Postprint available at: Linköping University Electronic Press

$\underline{\text { http://urn.kb.se/resolve?urn=urn:nbn:se:liu:diva-112560 }}$ 


\title{
Multiport Technology - the New Rise of an Old
}

\section{Concept}

\author{
S.O. Tatu, A. Serban, M. Helaoui, A. Koelpin
}

Abstract-A short review of the multiport (six-port) theory for modern applications in downconversion, direct demodulation or modulation of RF signals, and new measurement techniques for localization is presented in this paper. More than four decades ago, the circuit was developed for low-cost S-parameter measurements of microwave circuits. The modifications required to transform original six-port instrument function to radio function was not obvious in the beginning. In this purpose, several innovations have been implemented during the last two decades. Implementations of the circuit in various mature fabrication technologies, covering frequencies from microwave to millimeter wave spectrum, are presented and discussed. Multiport receiver calibration and impairment mitigation techniques are also proposed at frequencies where analog-to-digital converters and digital signal processing are available.

Index Terms - multiport technology, front-end, down-conversion, direct modulation, radar, angle of arrival, Six-Port.

\section{INTRODUCTION}

Modern society shows a big need for ubiquitous and wireless connectivity, information transmission with high data rates, and precise sensors for any kind of physical dimension. But frequency spectrum is limited and bandwidth restricted by regulatory issues. Over the last year a continuous rise in operating frequency can be observed linked to rising bandwidth. For short range operation, the license free industrial, scientific, and medical (ISM) bands beyond $100 \mathrm{GHz}$ get more 
and more into focus for practical applications. At the same time, also microwave monolithic integrated circuit (MMIC) processes must keep up with this rise in operating frequency what is associated with high investment costs in new MMIC technology. For the first years before this technology gets down to reasonable costs, alternative hybrid circuit concept are needed as a door opener for mass market applications. In this framework, an old front-end concept gets more and more supporters leading to a constant growth of an originally small but worldwide community: Multiport technology. This publication aims do give an overview over the basics, possible applications, as well as specific calibration procedures for the most common multiport implementation of this promising architecture: the six-port.

The multiport circuit theory was initially developed in the 1970's by scientists for accurate automatized measurements of the complex reflection coefficients, in microwave network analysis [1]-[3]. These multiport pioneers highlighted its usefulness in microwave low-cost circuit characterizations (S-parameters).

Since 1994, the multiport techniques were further developed for microwave and millimeter wave radios [4]-[6]. Several multiport architectures for specific applications such as communication transceivers, automotive radar, or imagery have been developed and implemented, until today. Basically, the multiport is a passive circuit composed of several couplers interconnected by transmission lines and phase shifters. Its specific architecture and design are strongly related to the target application and the operating frequency. The multiport acts as an interferometer; its output signals are linear combinations of phase shifted input signals. By using the appropriate circuit design and appropriate devices, connected to the output ports, this circuit can provide specific parameters, such as reflection coefficient, distance or modal measurements, phase and frequency analysis, quadrature down-conversion, or direct-carrier modulation of microwave /millimeter wave frequencies. 
As originally designed for automated measurements of the complex reflection coefficient, the multiport has a local oscillator input, a measurement port, and four outputs [3]. One of the outputs is used as a reference power level and powers measured at the other ones are function of the complex coefficient of the device under test, connected to the measurement port. There are three different reflection coefficient values, named $q_{\text {i }}$ points, which minimize the power at the corresponding $i$ output. The ideal architecture requests that $q_{\mathrm{i}}$ points are spaced by $120^{\circ}$ and located equidistant from the origin of the complex plane.

The new application fields require a different architecture of the circuit and new modules, to be connected at its ports. The S parameter matrix of the multiport circuit reveals that there are two clusters of ports, 1 to 4 and, 5 and 6 . Inside each cluster, all the ports are perfectly matched and isolated one versus the others [7]. In all applications they play separate functions, such as: four outputs and, respectively, two RF inputs for down-conversion, or four control inputs and RF output/input for direct modulators. If the $\mathrm{S}$ matrix is further analyzed, it is straightforward that if four matched loads are connected to the first group of ports (1 to 4) and two RF signals are applied to other pair of ports (5 and 6), all output signals at first group of ports are function of both input signals of the second group. This is a fundamental difference, if compared to the multiport used in reflection coefficient measurements, where one of the outputs is used as a power reference [3]. The multiport has now four $q_{\text {i }}$ points, spaced by $90^{\circ}$ multiples, and located equidistant from the origin of the complex plane. The phase difference between the pair of odd $q_{\text {i }}$ points is $180^{\circ}$. The same result is obtained for the pair of even points [8].

It is to be noted that same denomination of the multiport inputs and outputs are used trough this paper to avoid confusions for the reader, especially in equations. Therefore, the port numbers can be sometimes different if compared to certain references.

The use of multiport technology in RF design is a good choice, especially if the operating 
frequency is in the high microwave or millimeter-wave range. The dimensions of the multiport circuit fabricated in Miniature Hybrid Microwave Integrated Circuit (MHMIC) technology, usually around 1.5 times of the operated wavelength in square, become small enough to be integrated on the same substrate with antennas [9]. Even if the multiport is further miniaturized, the antenna or array antenna size will determinate the final dimensions of a front-end. The multiport circuit can be also used in the front-end design to operate at the frequencies where active components are not yet available in the market. In order to operate as demodulator or modulator, it requires only the use of

power detectors or switches. Therefore, research activities can be validated by front-end prototyping measurements, years before standard technologies become available.

This paper highlights, trough several examples and multiple references, the modern applications of the multiport technology, and significant advances in fabrication procedures, in the recent years.

\section{MultiPort Down-CONVERSION AND DiRECT DEMODUlation}

The simplified block diagram of a typical multiport down-converter is plotted in Fig. 1. The local oscillator (LO) is connected at port 5 and the low-noise amplifier (LNA) with antenna at port 6. Square-low matched power detectors are connected to the output ports 1 to 4 of the circuit. The output signals are linear phase shifted combination of inputs, as seen in the same figure. Two differential amplifiers are used, in order to obtain the output quadrature signals I and Q. The circuit can operate either in direct conversion or low intermediate frequency (IF) schemes. 


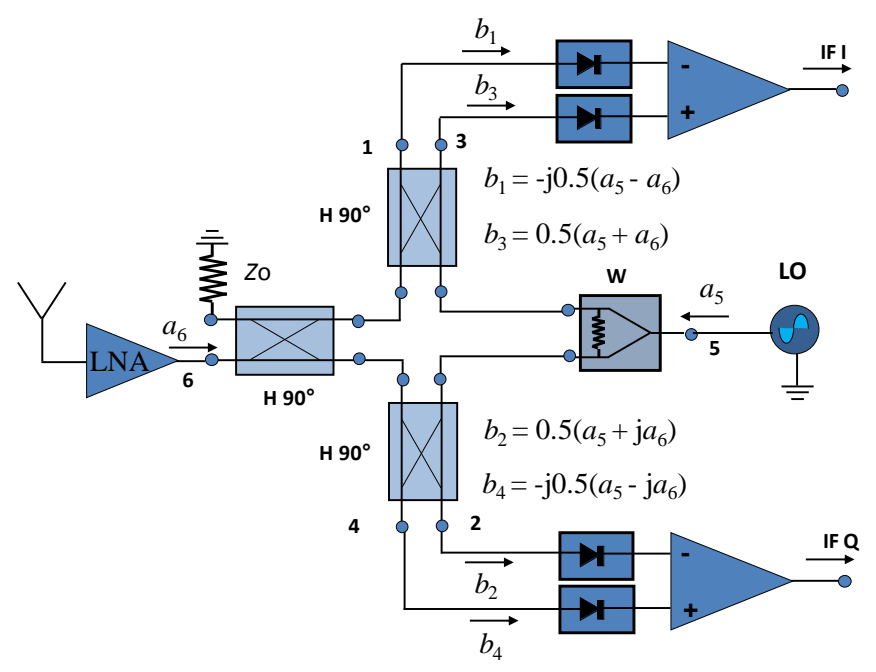

Fig. 1. The simplified block diagram of a multiport circuit for down-conversion.

If the RF input signal is amplitude modulated, the amplitude of quadrature signals are direct proportional to its amplitude. For a QAM signal, the response of the demodulator versus the amplitude and phase variation of the RF input signal is linear.

We note that, if the circuit is used as a low IF downconverter, a second in-phase down-conversion and lowpass filtering is required [7].

One of earlier implementations of this multiport module, a wide-band $(23-31 \mathrm{GHz})$ circuit has been fabricated in MHMIC technology, on a $254 \mu \mathrm{m}$ ceramic substrate with a relative permittivity $\varepsilon_{\mathrm{r}}=9.9$. The chip measures $25.4 \mathrm{x}$ $25.4 \mathrm{~mm}^{2}$. The complete RF microstrip topology is shown
Demodulator's

Input/Output Dependencies:

As presented in [7], if the inputs signals for $\mathrm{LO}$ and $\mathrm{RF}$ are

$$
\begin{gathered}
a_{5}=a e^{j\left(\omega_{0} t+\varphi_{5}\right)} \\
\text { and } \\
a_{6}=\alpha(t) a e^{j\left(\omega t+\varphi_{6}(t)\right)}
\end{gathered}
$$

the output IF signals read

$$
v_{I F}^{I}(t)=\alpha(t) K a^{2} \cos [(\omega-
$$

$\left.\left.\omega_{0}\right) t+\left(\varphi_{6}(t)-\varphi_{5}\right)\right]$

and $\quad v_{I F}^{Q}(t)=\alpha(t) K a^{2} \sin [(\omega-$ $\left.\left.\omega_{0}\right) t+\left(\varphi_{6}(t)-\varphi_{5}\right)\right]$

for inphase $I$ and quadrature $Q$ component.

The constant value $K$ is related to power detectors' efficiency and differential amplifiers' gain.

Previous equations show that the circuit can act also as a direct demodulator $\left(\omega=\omega_{0}\right)$.

in Fig. 2 and consists of a wide-band Ka band multiport junction with surface-mounted RF diodes and related matching circuit network [10]. 


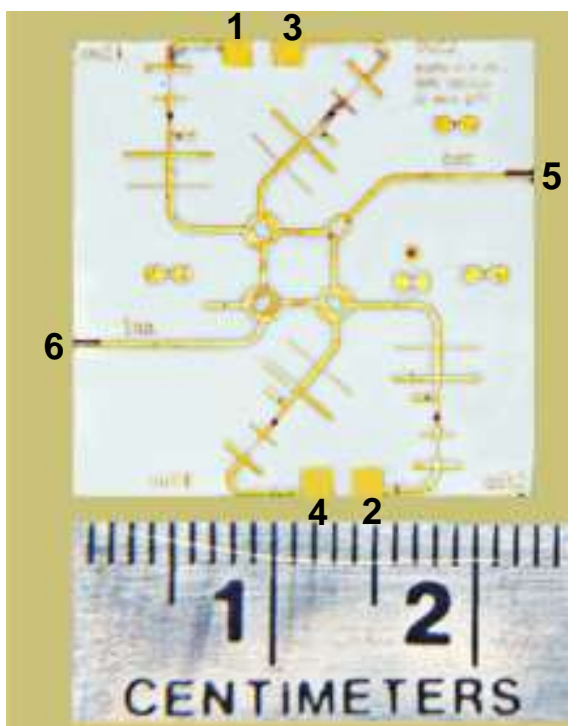

Fig. 2. Photograph of the Ka-band MHMIC multiport module.

The demodulation results of the circuit have been presented in details in [11]. A snapshot of direct demodulation results of usual PSK/ QAM signals, using the MHMIC multiport circuit previously described, is illustrated in Fig. 3. The images are captured using a digital oscilloscope in XY format, using a color grade plot. The LO power level is set at around $-25 \mathrm{dBm}$ and the data-rate at 20 Msym/s.

In the last decade, the operating frequency has been pushed up to millimeter wave spectrum [8], [9], [12]. The multiport circuit is always composed by four couplers, which can be $90^{\circ}$ hybrids, power detectors, or rat-race ones. The choice of the couplers is related to the designer experience, operating frequency, required size, and shape of the circuit [6]. 


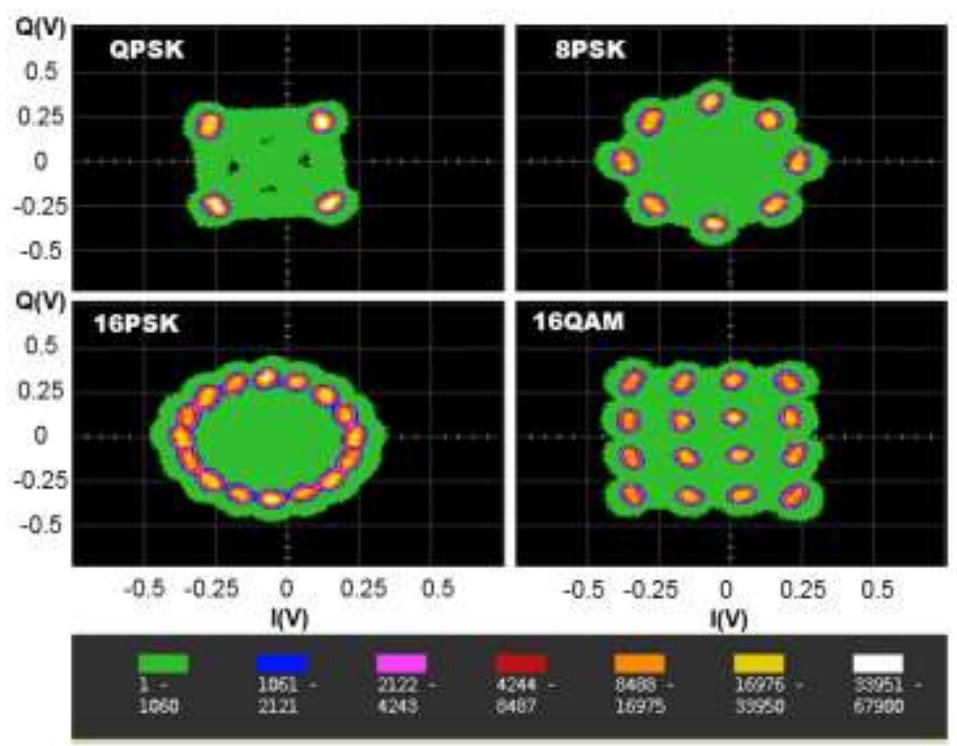

Fig. 3. Demodulation result of usual PSK/QAM signals using color grade display.

The main advantage of using the multiport module in down-conversion or direct demodulation is the reduced LO power to operate. As suggested by the equations (1) to (4), the use of power detectors require comparable power levels at LO and RF ports. This is an important advantage, especially in millimeter waves, where power levels are more difficult to generate. In addition, due to limited insulation between RF inputs, it helps in direct conversion, to reduce DC offset.

The same technique can be used in the near future at the $\mathrm{THz}$ frontier, and requires only the use of power detectors.

\section{Multiport Direct Modulation}

On the transmitter side of a communication system, the multiport technology can be used to implement a variety of high data rate modulation schemes, such as QPSK and M-QAM [13]-[19]. The modulator hardware complexity is reduced by combining the modulation and up-conversion in a direct-carrier modulation scheme.

The simplified block diagram of a typical multiport direct modulator is shown in Fig. 4. The LO signal represented as the incident wave $a_{6}$ is applied to port 6. Ports 1, 3, 2 and 4 are connected at 
terminal impedances $Z_{\mathrm{i}}(i=1$ to 4$), Z_{\mathrm{i}} \neq Z_{0}$, where $Z_{0}$ is the system impedance. The LO signal $a_{6}$, experiences different phase shifts and attenuations when it passes the six-port correlator. Reaching the loads at ports $1,3,2$, and $4, a_{6}$ is reflected with different reflection coefficients $\Gamma_{1}, \Gamma_{3}, \Gamma_{2}$, and $\Gamma_{4}$ by the terminal impedances $Z_{i}$, where $\Gamma_{i}=\left(Z_{i}-Z_{0}\right) /\left(Z_{i}+Z_{0}\right)$. The reflected waves are finally summed by the Wilkinson power divider $(\mathrm{W})$ at port 5 .

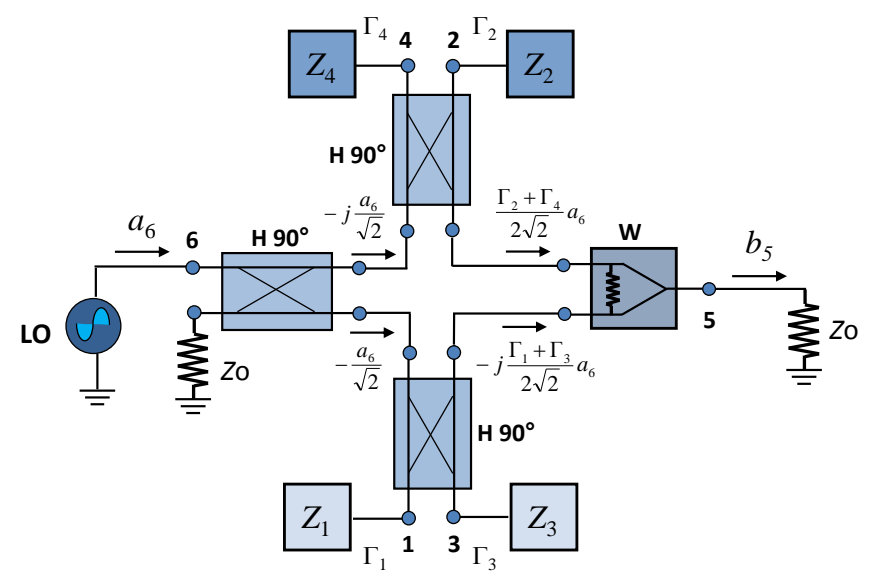

Fig. 4. The simplified block diagram of a multiport circuit for direct carrier modulation of a RF signal.

In order to implement M-QAM modulation schemes, the reflection coefficients $\Gamma_{\mathrm{I}}$, and $\Gamma_{\mathrm{Q}}$, have to be varied in their values, accordingly to the modulating I- and Q- baseband signals. With this assumption, the interpretation of (6) becomes that $a_{6}$ carrier signal shifts the spectrum of the baseband signal $\Gamma_{\mathrm{I}}(\mathrm{t})+j \Gamma_{\mathrm{Q}}(\mathrm{t})$ from baseband up to the carrier frequency, $\omega_{\mathrm{LO}}$, hence, the modulated RF signal $v_{\mathrm{RF}}(\mathrm{t})$.

Six-Port Modulator Dependencies:

Due to the phase relations in the six-port correlator and depending on the reflections taking place at the load impedances, the ideal output signal at port $5, b_{5}$ can be written as:

$b_{5}=-\frac{a_{6}}{4}\left[\left(\Gamma_{1}+\Gamma_{3}\right)+\right.$
$\left.j\left(\Gamma_{2}+\Gamma_{4}\right)\right]$

It can be observed that $\left[\Gamma_{1}+\Gamma_{3}+\right.$ $\left.\mathrm{j}\left(\Gamma_{2}+\Gamma_{4}\right)\right]$ in (5) has the form of a complex envelope of any quadrature modulated signal.

Assuming modulator encoding schemes with $\Gamma_{1}=\Gamma_{3}=\Gamma_{\mathrm{I}}(\mathrm{t})$, and $\Gamma_{2}$ $=\Gamma_{4}=\Gamma_{\mathrm{Q}}(\mathrm{t})$, and expressing the carrier $a_{6}$ in phasor form, (5) becomes:

$v_{\mathrm{RF}}(\mathrm{t})=-\frac{A_{L O}}{2}\left[\Gamma_{\mathrm{I}}(\mathrm{t})+\right.$ $\left.j \Gamma_{\mathrm{Q}}(t)\right] e^{j\left(\omega_{L O} t+\varphi_{L O}\right)}$

where $A_{\mathrm{LO}}$ is the amplitude, $\omega_{\mathrm{LO}}$ is the angular frequency and $\varphi_{\mathrm{LO}}$ is the relative phase of the LO signal. 
Controlled impedance values can be implemented by means of RF switches under digital signal processing (DSP) control in conjunction with passive impedance terminations, e.g., impedances corresponding to SHORT and OPEN conditions [19]. However, for higher data rates, M-level quadrature amplitude modulation (M-QAM, $\mathrm{M}=16$, 64, etc.) are necessary. Therefore, more than two impedance termination levels must be generated and controlled. Active impedance terminations implemented with field-effect transistors (FETs) and with Schottky diodes have been firstly proposed [13]-[14].

Prototypes for direct-carrier six-port modulators using FETs and Schottky diodes for impedance generation were designed and fabricated for a center frequency of $7.5 \mathrm{GHz}$. In Fig. 5, the photograph of the multiport modulator with Schottky diode HSMS286Y from Avagotech, manufactured on a Rogers $4350 \mathrm{~B}$ substrate with a thickness of $0.254 \mathrm{~mm}$, is shown.

The modulation results of the circuit have been presented in details in [14] and are illustrated in Fig. 6.

The main advantages of using the multiport (six-port) module in direct modulation are becoming evident when these ones are compared to conventional mixer based up-conversion modulators [13][18].

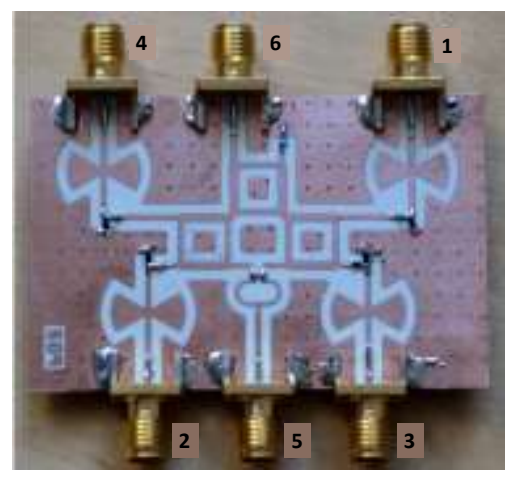

Fig. 5. Photograph of the ROGER 4350B multiport modulator, with Schottky diodes [14]. 


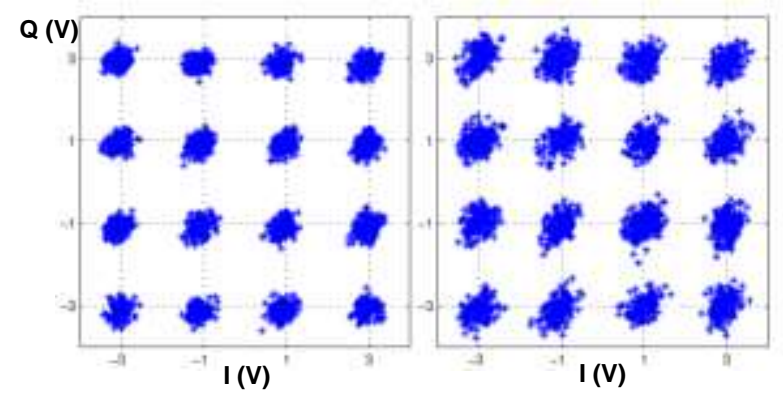

Fig. 6. Direct-carrier modulation result using the circuit of Fig. 5: (a) $100 \mathrm{Msym} / \mathrm{s}$ and (b) $300 \mathrm{Msym} / \mathrm{s}$, [14].

As seen previously, the multiport modulator is a linear circuit using passive components that generates the modulated RF signal directly from an LO signal in terms of the reflection coefficients. The input LO-power is mostly related to the output RF-power, as illustrated by (6). This relationship gives the possibility to directly control the level of the output RF signal through the LO input power and, eventually, to avoid the use of power-hungry and high-complexity power amplifiers (PAs). This is an interesting aspect for many short-range applications requiring high-data rates in GHzrange with low transmitted power.

In contrast, conventional mixers' based modulators (e.g., Gilbert cell), operate in switching mode to achieve the required up-conversion, hence they require a certain level of LO power. Moreover, the conventional up-conversion mixers are narrowband, but the multiport direct-carrier modulators can be designed for broadband operations.

Supplementary, the analog/RF processing of signals by means of simple microwave techniques, as those presented in [15]-[17], has proven the diminution of LO-leakage, which represents a typical problem for direct conversion radio architectures resulting in DC-offsets. 


\section{MultiPORT FOR LOCALIZATION}

Multiport technology is not only capable for data transmission and network analysis, but can be also used for localization purposes [20]. Besides distance measurements, angle-of-arrival (AoA) and vibration analysis are also promising applications [21].

Localization by multiport technology is based on the high phase resolution of the front-end, that can be in general constructed as shown in section II, and completed to a radar system, as can be seen in Fig. 7 for the six-port architecture.

In addition to the six-port circuitry, a hybrid coupler is used to split a part of the synthesizer's transmit signal as a phase reference to one of the six-port inputs, and, also, to couple the received signal, scattered back by the target, to the other sixport's input port. If the signal frequency $f_{\mathrm{RF}}$ is Dependency between distance and phase: $d=\frac{x}{2}=\Delta \varphi \cdot \frac{c}{2 \cdot 2 \pi \cdot f_{R F}}$ In the formula, $c$ represents the propagation velocity and $x$ the total run length of the signal to the target, and back to the antenna.

The phase $\Delta \varphi$ can be calculated from the baseband voltages $b_{1}$ to $b_{4}$ according to

$$
\Delta \varphi=\tan ^{-1}\left(\frac{\jmath m}{\mathcal{R} e}\right)=\tan ^{-1}\left(\frac{b_{4}-b_{2}}{b_{3}-b_{1}}\right)
$$

known, the distance $d$ between antenna and target

can be reconstructed according to (7). The path difference between reference and received signal is represented by the phase difference $\Delta \varphi$ between both signals and, therefore, easily detectable by a six-port network.

As you can see from (7) $f_{\mathrm{RF}}$ has to be known with high accuracy, for high resolution distance measurements. Therefore, most multiport based radar front-ends use well defined continuous wave $(\mathrm{CW})$, or two or more different $\mathrm{CW}$ frequencies, as transmitted signal. As for common $\mathrm{CW}$ radar, the distance measurement values for the multiport radar are also ambiguous in $2 \pi$. This results from the obvious fact that for only one frequency you will not be able to distinguish in which period of the signal the measured phase difference, i.e. the position of the target, will occur. On the other hand, this instance motivates the very high distance resolution of the multiport radar, since the total 
dynamic range of the system is mapped to the distance of only one wavelength, and not scaled to the total distance. Values in the micrometer range for resolution can be obtained at Ka-band frequencies, with state-of-the-art analog to digital converters (ADC) [21].

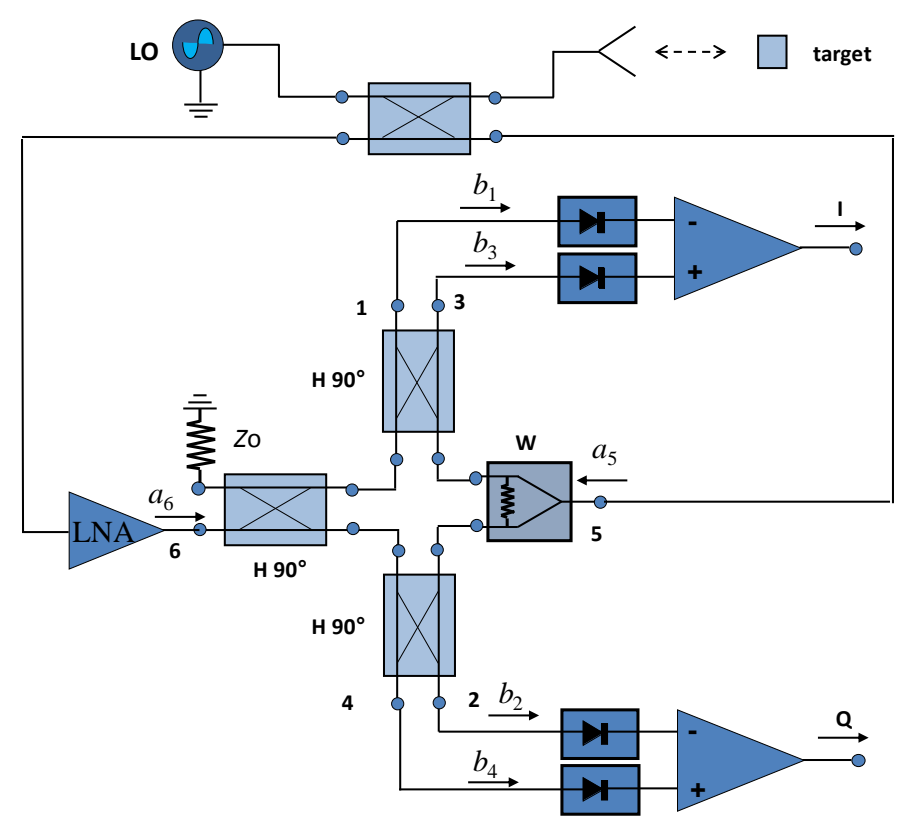

Fig. 7. Radar front-end for distance measurements based on six-port technology.

The ambiguity issue - well known for common CW radar - can be solved by using two or more different frequencies: if you measure a static target scenario with different frequencies, you will end up with different relative phases, as a different number of periods for the different wavelengths will fit in the distance between antenna and target. The phase dependency between both signals is unique up to a distance equivalent to the wavelength of the beat frequency, i.e. the difference frequency, of the two signals, respectively. By this trick, the ambiguity free measurement distance can be expanded to the wavelength of the resulting beat frequency. Using more than two different frequencies will result in high unambiguous ranges.

The phase of the beat frequency shows comparably low distance resolution, due to superposition of non-idealities from both signals and other effects. Therefore, the multi-tone signal is only used to 
determine the actual period of the signal for the highest used frequency $f_{\max }$. This offset can be added as a multiple of $2 \pi$, to eliminate the phase ambiguity at $f_{\max }$.

For precise ultra-high resolution measurements in the one-digit micrometer or nanometer range, all effects arising from the front-end and the environment have to be determined and compensated (e.g. frequency dependency of the diode detectors and the antenna's phase center, as well as the detectors' power characteristics, influence of temperature and moisture on propagation velocity in the radar channel, and, of course, temperature drift and aging of all components of the front-end).

Compared to common radar technologies, multiport radar systems benefit from the very low complexity concerning algorithms for distance reconstruction from the baseband output voltages of the multiport structure. For six-port architecture, the signal processing is minimized to only determine the argument of a complex valued number, represented by the differential I/Q baseband voltages $b_{1}$ to $b_{4}$. The microcontroller only has to calculate the quotient of the voltage values for imaginary and real part, build the arctangent of this relation, as shown in (8), and use the value for $\Delta \varphi$ to evaluate the distance $d$ in (7).

The distance value $d$ can be calculated by only few clock cycles of the processor, resulting in very low latency and as no long lasting algorithms (e.g. if high length FFT are used, high measurement value update rates can be realized). In general, the speed defining component will be the ADC, with its limited sampling rate.

From the high update rate of the system, further applications arise besides static target distance measurement scenarios: multiport systems for vibration monitoring based on distance measurements with high update rate. It has been shown that a six-port based radar system can be used as a radar microphone analyzing the movement of a loudspeaker membrane [22], or even detecting the heartbeat and breathing rate of a person [23]. The big advantage of high resolution distance analysis compared to common Doppler radar approaches is that also very low velocities up to zero 
movement can be detected as well as aperiodic and pulse-like movements. This concept is very promising and can be also used in industrial applications where modal analysis is a well-known tool to monitor process conditions [24].

Besides distance measurements, Angle of Arrival (AoA) analysis can be also used for localization purposes. Multiport technology offers a very simple and highly accurate tool for detecting the direction of an impinging wave with millidegree resolution.

There are two basic concepts using six-port and multiport receivers for AoA:

(i) Several parallel receivers for coherent signal reception as for common receiver architectures: the impinging wave is received and down-converted by a local oscillator's signal, that is distributed phase compensated to all receiver modules. The AoA analysis is conducted by baseband processing as for common receiver systems.

(ii) One (besides LNAs) full passive six-port or multiport front-end with two or more antennas.

For the second approach, multiport technology offers a very simple and precise method that will be explained here in detail.

The signal is received by two antennas, optionally amplified and fed to the two input ports of the six-port structure, as seen in Fig. 8. The relative phase difference of the received signals due to their different path lengths is mapped to the power of the four output signals, down-converted and digitized. From these values, the AoA $\alpha$ can be easily calculated by (9) using the distance $v$ between the antennas, baseband voltages $b_{1}$ to $b_{4}$, and the wavelength $\lambda$ of the received signal [25]. 


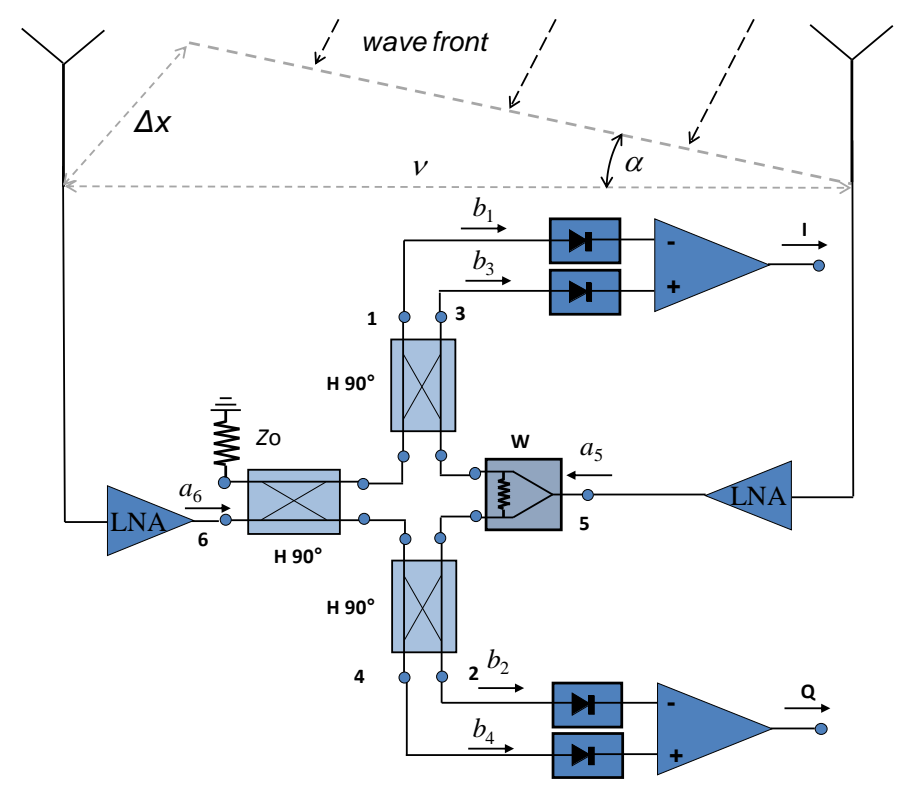

Fig. 8. Angle-of-Arrival detector based on one six-port system.

As for the distance measurements, there is also an ambiguity for AoA detection. The ambiguity range depends on the distance $v$ between the antennas. The larger the distance, the higher the angular resolution, and

Dependency between AoA and phase: $\alpha=\sin ^{-1}\left[\frac{\lambda \cdot \tan ^{-1}\left(\frac{b_{4}-b_{2}}{b_{3}-b_{1}}\right)}{2 \pi \cdot v}\right]$ where $v$ represents the antennas' distance.

the smaller the un-ambiguous region are. This dependency is explained in detail in [26].

Similarly to the multi-tone concept for solving the ambiguity issues for ranging, a similar concept can be applied to scale the ambiguity free range for AoA: the dual six-port approach. For this solution, another six-port receiver is added, having the antennas in the same geometric dimension as for the first six-port system, but featuring a different antenna distance $v$ [26]. This leads to different periodicity for both systems, as can be observed in Fig.9. 


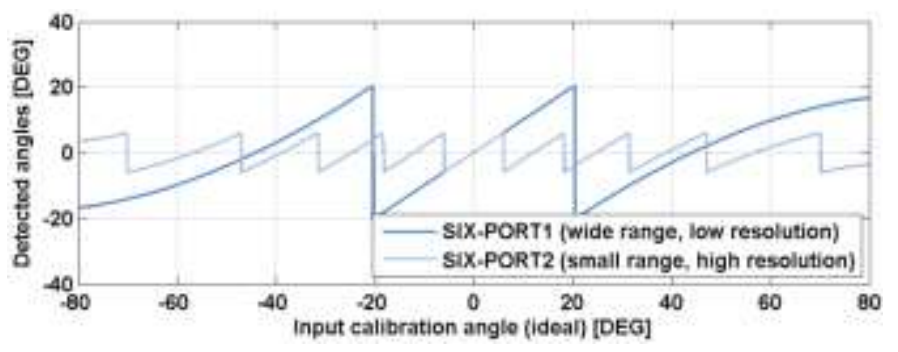

Fig. 9. Phase ambiguity for two different antenna distances $v_{1}$ for six-port 1 and $v_{2}$ for six-port 2 .

The combination of the periodicity for both antenna distances is unique for each AoA and, therefore, unambiguous for a much larger range of angles, as for both distances alone.

In conclusion, you can solve the AoA ambiguity by analyzing the phases for both six-port systems, and the phase difference between both results. From this difference, the absolute ambiguity period can be calculated. This can be added as an equivalent angular offset to the more precise AoA value, gained from the larger antenna distance.

As for common continuous wave (CW) radar systems, six-port or multiport radar is, in its simplest setup, not capable of multi-target scenarios and multi-path effects. Parasitic reflection signals from other targets or delayed multi-paths will be superimposed to the original signal, leading to an error. It is important to keep in mind that this error cannot be detected or compensated, and the resulting measured value will be the mean value of all received phases, weighted by their signal power, respectively. For static scenarios and the possibility of initial teaching steps of the target, some of these phase offsets can be eliminated up to a certain degree. For dynamic parasitic behavior, this is not possible, and other countermeasures have to be considered.

\section{Multiport Wideband Receiver Calibration AND ImPairment Mitigation}

The architectures shown in Fig. 1, Fig. 4 and Fig. 5 are simple to implement since they provide direct access to the in-phase $(I)$ and quadrature $(Q)$ components of the complex envelope of the 
modulated signal. They don't involve any calibration or impairment compensation before getting to the signal's complex envelope detection. The use of un-calibrated multiport receivers assume that the gains through the two paths RF inputs to $b_{1}$ and $b_{3}$ (similarly between RF inputs to $b_{2}$ and $b_{4}$ ) are quasi identical and also that the phase shifts are multiple of $90^{\circ}$, as demonstrated in multiport theory [7]. This approach involves a very accurate design of multiport circuits over the entire frequency band of operation and cannot be avoided for low power applications or applications with high datarates that surpass the limits of today's DSP.

If technical conditions, required applications, and cost permit, calibration procedures can considerably improve the demodulation results. This is particularly true for wireless communication applications where complex modulation schemes and frequency multiplexing techniques are used. Such applications require accurate demodulation and a receiver that operates at frequencies away from the central operating frequency where the circuit has been designed. Besides, the calibration techniques allow to compensate for S-parameter degradation due to fabrication process errors. Moreover, power detectors have limited dynamic range of the square law region, which results in an overall poor signal-to-noise ratio of the receiver. To extend this dynamic range, one should operate the power detectors beyond this region and close to their saturation, where the proportionality between the output voltage and RF power is not perfectly maintained. These imperfections generate distortion in the reconstructed complex envelope of the signal unless distortion compensation algorithms such as post-distortion techniques are used to linearize the power detectors behavior.

In order to calibrate the six-port circuit drift from ideal conditions and to linearize the power detectors, access to the output of each power detector is required. Therefore, a modified architecture of a six-port receiver was proposed in [27], [28] for broadband receiver applications.

Fig. 10 shows a block diagram of the proposed architecture. The output of each power detector is digitized with an ADC and passed to a DSP where the calibration and post-distortion algorithm is 
carried out, before the generation of the complex envelope in-phase and quadrature components of the received signal.

\section{A. Calibration for Non-ideal Six-port}

\section{circuit}

In the case of non-ideal conditions, it can be shown [28] that the power reading from each of the four power detectors is a linear combination of a constant, the in-phase component, the quadrature component, and the complex envelope of the receiver signal. This relationship is summarized in (10). Therefore, by solving the linear system, one can express the in-phase and quadrature components as a linear combination of the four power readings from the power detectors. The operation of solving for the coefficients $\alpha_{i}$ and $\beta_{i}$ required to demodulate the received signal is called linear calibration, where the 'linear' term is inherited from the linear system of equation that needs to be solved during the operation. While the data samples and therefore power reading change versus time, the coefficients should be constant theoretically and finding these

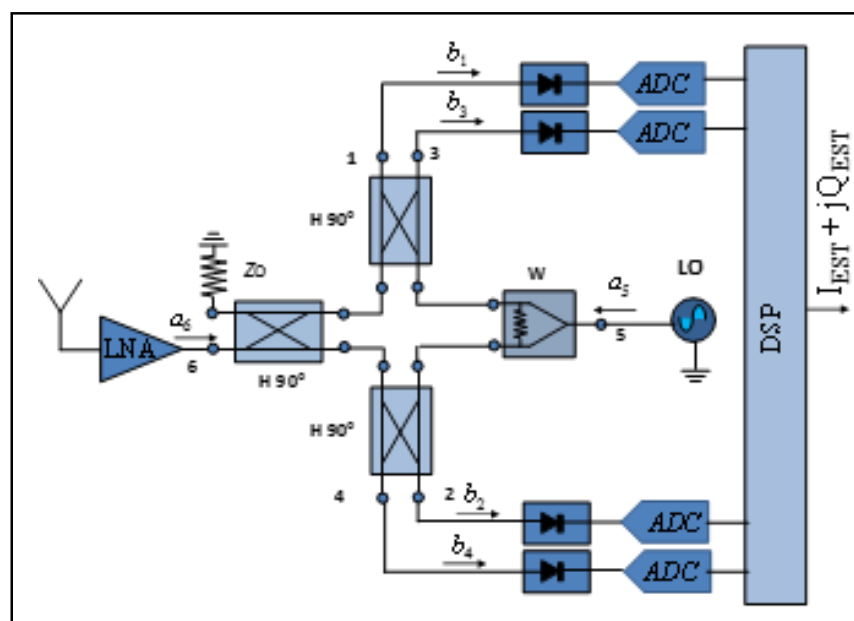

Fig. 10. Block diagram of a six-port receiver suitable for wideband applications.

Linear Calibration in Non-ideal Six-port Receivers:

\section{- Relationship between the power readings and in-} phase and quadrature data

$\left[\begin{array}{l}P_{1} \\ P_{2} \\ P_{3} \\ P_{4}\end{array}\right]=\left[\begin{array}{llll}T_{11} & T_{12} & T_{13} & T_{14} \\ T_{21} & T_{22} & T_{23} & T_{24} \\ T_{31} & T_{32} & T_{33} & T_{34} \\ T_{41} & T_{42} & T_{43} & T_{44}\end{array}\right]\left[\begin{array}{c}1 \\ I_{R F}^{2}+Q_{R F}^{2} \\ I_{R F} \\ Q_{R F}\end{array}\right]$

where the matrix $[T]_{4 \times 4}$ coefficients are dependent only on the $S$-parameters of the sixport junction and of the LO signal amplitude and phase.

\section{- Linear calibration equation:}

From (10), $I_{R F}$ and $Q_{R F}$ can be written as a linear combination of $P_{1}, \ldots, P_{4}$ by inverting $[T]_{4 \times 4}$ matrix.

$$
\begin{aligned}
& I_{R F}=\sum_{i=1}^{4} \alpha_{i} P_{i} \\
& Q_{R F}=\sum_{i=1}^{4} \beta_{i} P_{i}
\end{aligned}
$$

Coefficients $\alpha_{i}$ and $\beta_{i}$ are constants that form the third and fourth row of $[T]_{4 \times 4}^{-1}$ matrix, respectively. 
constants for one known sample can be used with other samples as well.

However, in practice, the power readings include circuits' noise and impairments, which may affect the calibration process. One can reduce the effect of noise by using a known training sequence with a set of different samples for the purpose of calibration [28], [29]. The coefficients can then be estimated using a calibration algorithm based on least square estimation.

\section{B. Post-compensation of Power Detectors}

In order to increase the dynamic range of the six-port receiver, the diodes used for power detection are operated in and beyond their square law region. Therefore, their static nonlinear behavior is not ideal and can be reverse modeled using a memoryless polynomial function relating the RF power $P_{i}$ to the detected voltage $v_{i}$ as detailed in [30], [31]. This static memoryless polynomial model does not take into account any memory effect. Recently, it was shown that in wideband applications, the diodes exhibit memory effect that should also be taken into account when compensating for their impairments [31], [32].

Taking into account this memory effect, the diodes reverse model can be modified from a static memoryless polynomial to a memory polynomial that includes the dynamic nonlinearity. The expression of this model is given in (12) [28], [31]. The calibration and linearization of the six-port receiver can then be done in two steps:

1. The first step consists of power detectors linearization. For each power detector, the coefficients of the models of (12) are extracted using a known training sequence. The model is then applied to the estimate the RF power at the input of that power detector from its output voltage reading.

2. The second step is the calibration. All the estimated four power values are used to extract the estimated in-phase and quadrature components of the received signal as shown in (11). 
However, calibrating the six-port receiver and linearizing the power detectors in two different steps is not practically feasible as the system can be already integrated with no possibility to access the exact values of the RF powers necessary for six-port calibration. In addition, for wideband applications, one can assume that six-port circuits may also exhibit memory effects in terms of linear frequency response. Therefore, a joint calibration and post compensation model is required for complete compensation of the six-port receiver imperfections.

\section{Joint Calibration and Post-compensation Model}

By combining (11) and (12) and by assuming that the frequency response of the six-port circuit can be combined with the memory effect compensated for in the power detectors, one full model can be obtained for the joint compensation of impairments in the six-port circuits as well as the power detectors dynamic distortions. This model is detailed in (13).

A detailed block diagram is therefore given in Fig. 11, where the outputs of the sixport circuit of Fig. 10 are connected to detectors ADC and a DSP unit for calibration and impairment compensation.

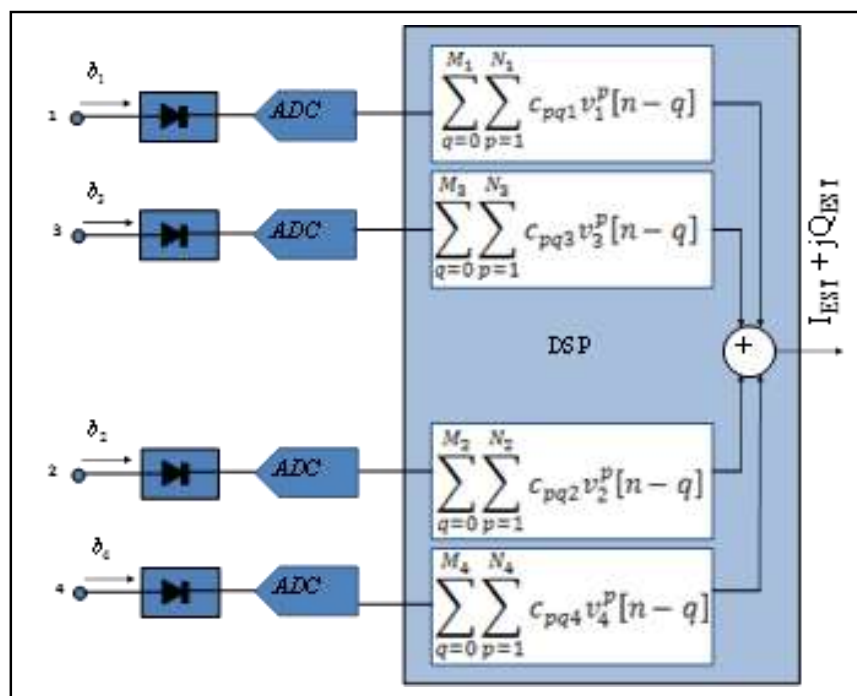

Fig. 11. Detail of signal processing block for calibration and impairment compensation for the receiver of Fiq.10.

A memory polynomial reverse modeling of the power detector allowing to obtain an estimate of the RF power $P_{i}$ to the detected voltage $v_{i}$ in a power detector, is given by:

$$
P_{i n, i}[n]=\sum_{q=0}^{M_{i}} \sum_{p=1}^{N_{i}} c_{p q i} v_{i}^{p}[n-q]
$$

where $c_{p q i}$ are the coefficients of the model, $N_{i}$ is the non-linearity order, and $M_{i}$ is the memory depth for the diode detector $D_{i}$.

\section{- Joint calibration and post-compensation}

From (10), $I_{R F}$ and $Q_{R F}$ can be written as a linear combination of $P_{1}, \ldots, P_{4}$ by inverting $[T]_{4 \times 4}$ matrix.

$\left(I_{E S T}+j Q_{E S T}\right)[n]=$

$\sum_{i=1}^{4} \sum_{q=0}^{M_{i}} \sum_{p=1}^{N_{i}} \gamma_{p q i} v_{i}^{p}[n-q]$

where $\gamma_{p q i}$ are the complex coefficients of the model. 


\section{Study: Multi-standard Six-port Receiver for Wireless Communications}

A six-port receiver using commercial coaxial components (three quadrature couplers and one Wilkinson combiner) was built to cover $2-3 \mathrm{GHz}$ band. It is known that such commercial components have relatively poor performances compared to a carefully designed multiport for a specific band and application.

Using above calibration procedures, we will demonstrate an important improvement of demodulation results for this specific receiver.

The six-port was measured, calibrated, and evaluated for different carrier frequencies, different signal standards, modulations, and bandwidths. For consistency of the results, the same order of nonlinearity $\left(N_{i}=3\right)$ and memory depth $\left(M_{i}=9\right)$ were used for all the four power detectors and all the types of signals.

The results showing the performance of the receiver in terms of EVM are summarized in Table I. The measured results showed that the performance of the receiver without calibration is deteriorating as a function of the bandwidth increase if no calibration and post-distortions are applied. These corrections techniques when applied bringing down the EVM to acceptable levels even for wideband signals with high dynamic range such as the $10 \mathrm{MHz}$ WLAN signal, where the EVM of the received signal is reduced to $3.4 \%$.

\section{CONCLUSION}

Through several well chosen examples, the paper highlights the multiport technology modern applications: down-conversion, direct demodulation or modulation of RF signals, and new measurement techniques for localization.

Its physical symmetry allows reduced amplitude and phase unbalancements over a wide frequency band. However, in the last 40 years, since the multiport (six-port) circuit is used, dozens of architectures have been proposed. The operating frequencies, fabrication technologies, and 


\begin{tabular}{|rcccc|}
\hline \multicolumn{5}{c|}{ TABLE I } \\
Measured & Performance of a Wideband Six-port Receiver \\
\hline Signal Type & $\begin{array}{c}\text { BW } \\
(\mathbf{M H z})\end{array}$ & $\begin{array}{c}\text { Carrier Freq. } \\
(\mathbf{G H z})\end{array}$ & $\begin{array}{c}\text { (EVM, \%) } \\
\text { No correction }\end{array}$ & $\begin{array}{c}\text { (EVM, \%) } \\
\text { With correction }\end{array}$ \\
\hline 64-QAM & 2 & 2.5 & 8 & 1.7 \\
64-QAM & 4 & 2.5 & 16 & 2.8 \\
WCDMA & 3.84 & 2.14 & 22 & 4.7 \\
WLAN & 10 & 2.4 & 20 & 3.4 \\
\hline
\end{tabular}

applications do not have ceased to evolve.

The paper demonstrates that multiport technology is a good competitor of the conventional approaches, especially in the microwave and millimeter-wave frequencies, where circuit dimensions, closely related to the guided wavelength, are in the $\mathrm{cm}$ range or smaller. Broadband circuits can be designed to cover a wide frequency spectrum and to allow quasi-optical data rates for wireless communication. Impressive resolution and high accuracy can be obtained in range or angle of arrival measurements. Calibration and impairment mitigation techniques can also be used to improve demodulation results, especially in low microwave spectrum, where ADC and DSP techniques are available today.

Future works target, among others, improvements in multiport circuit design for each specific application, the increasing of the operating frequencies, and, why not, the use of multiport systems in $\mathrm{THz}$ frequency range.

Multiport technology offers a promising opportunity for communications and wireless sensing at the leading edge of technology. This is the reason why a new rise of this old general concept can be observed over the last years. Maybe multiport technology also offers you a low-complex and interesting solution to your millimeter wave problems? 


\section{REFERENCES}

[1] S. B. Cohn, N. P. Weinhouse, "An automatic microwave phase measurement system," Microwave Journal, vol. 7, no. 2, pp. 49-56, 1964.

[2] G. F. Engen, C.A. Hoer, “Application of an Arbitrary 6-Port Junction to Power-Measurement Problems", IEEE Transactions on Instrumentation and Measurement, IM-21, pp. 470-474, 1972.

[3] G. F. Engen, "The Six-Port Reflectometer an Alternative Network Analyzer", IEEE Transactions on Microwave Theory Techniques, MTT-25, pp. 1075-1077, 1977.

[4] Ji Li., R.G. Bosisio, Ke Wu , "Computer and Measurement Simulation of a New Digital Receiver Operating Directly at Millimeter-Wave Frequencies", IEEE Transactions on Microwave Theory and Techniques, vol.43, no.12, pp.2766-2772, December 1995.

[5] T. Hentschel, “The Six-Port as a Communications Receiver”, IEEE Transactions on Microwave Theory Techniques, vol. 53, no. 3, March 2005.

[6] R.G. Bosisio, Y.Y. Zhao, X.Y. Xu, S. Abielmona, E. Moldovan, Y.S. Xu, M. Bozzi, S.O. Tatu, J.F. Frigon, C. Caloz, Ke Wu, “New-Wave Radio”, IEEE Microwave Magazine, pp. 89-100, February 2008.

[7] S.O. Tatu, E. Moldovan, S. Affes, "Multi-Port Front-End and Transceivers for V-Band MultiGigabit/s Communication Systems", Digital Front-End in Wireless Communications and Broadcasting Circuits and Signal Processing, ISBN:9781107002135, Chapter DOI: http://dx.doi.org/10.1017/CBO9780511744839.025, pp. 707-732, Cambridge University Press, Cambridge, UK, September 2011.

[8] T. Jiang, D. Hammou, C. Hannachi, J-F. Frigon, Ke Wu, M. Nedil, S.O. Tatu "Six-port Technology for Millimeter-wave MIMO Systems”, 2013 European Microwave Week, Conference Proceedings, pp. 1591-1594, Nuremberg, October 6-11, 2013. 
[9] S.O. Tatu, B. Boukari, E. Moldovan, R. G. Bosisio, Ke Wu, "Millimeter-wave Multi-port Radar Sensor with Integrated Receiver Front-end for Automotive Applications", 2012 IEEE Microwave Theory and Techniques Symposium, Conference Proceedings, ISBN: 978-1-46731088-8/12@2012 IEEE, Montreal, Canada, June 17-22, 2012.

[10] S. O. Tatu, E. Moldovan, Ke Wu, R.G. Bosisio, “A New Direct Millimeter-Wave Six-Port Receiver", IEEE Transactions on Microwave Theory and Techniques, vol.49, no.12, pp. 2517$2522,2001$.

[11] S.O. Tatu, E. Moldovan, Ke Wu, R.G. Bosisio, T. Denidni, "Ka-Band Analog Front-end for Software Defined Direct Conversion Receiver", IEEE Transactions on Microwave Theory and Techniques, vol.53, no.9, pp. 2768-2776, September 2005.

[12] S.O. Tatu, E. Moldovan, "V-band Multi-port Heterodyne Receiver for High-speed Communication Systems", EURASIP Journal on Wireless Comm. and Networking, Article ID 34358, 7 pages, 2007.

[13] A. Serban, J. Östh, M. Karlsson, Owais, S. Gong, J. Haartsen, P. Karlsson, "Six-Port Transceiver for 6-9 GHz Ultra-Wideband Systems," Microwave and Optical Technology Letters, vol. 52, no. 3, pp. 740-746, Mar. 2010.

[14] J. Osth, Owais, M. Karlsson, A. Serban, S. Gong "Schottky Diode as High-Speed Variable Impedance Load in Six-port Modulators," Ultra-Wideband (ICUWB), 2011 IEEE International Conference on, vol. 1, no. 4, pp. 68-71, 2011.

[15] J. Osth, A. Serban, M. Karlsson, S. Gong, "LO Leakage in Six-Port Modulators and Demodulators and its Suppression Techniques," 2012 IEEE/MTT-S International Microwave Symposium Digest, 2012, vol. 2, no. 4, pp. 1-3. 
[16] A. Serban, M. Karlsson, J. Osth, Owais, S. Gong, “Differential Circuit Technique for Six-Port Modulator and Demodulator," 2012 IEEE/MTT-S International Microwave Symposium Digest, vol. 5, pp. 6-8, 2012.

[17] J. Osth, M. Karlsson, A. Serban, S. Gong, “Carrier Leakage Suppression and EVM Dependence on Phase Shifting Network in Six-Port Modulator," Microwave and Millimeter Wave Technology (ICMMT), International Conference on, pp. 12-15, 2012.

[18] J. Osth, M. Karlsson, A. Serban, S. Gong, "M-QAM Six-Port Modulator Using Only Binary Baseband Data, Electrical or Optical," IEEE Transactions on Microwave Theory and Techniques, vol. 61, no. 6, pp. 2506-2513, Jun. 2013.

[19] Y. Zhao, J.-F. Frigon, Ke Wu, R. G. Bosisio, "Multi (Six)-Port Impulse Radio for UltraWideband," IEEE Transactions on Microwave Theory and Techniques, vol. 54, no. 4, pp. 17071712, Jun. 2006.

[20] A. Koelpin, G. Vinci, B. Laemmle, D. Kissinger, and R. Weigel, "The Six-Port in Modern Society", IEEE Microwave Magazine, vol.11, no.7, pp.35-43, Dec. 2010.

[21] G. Vinci, S. Lindner, F. Barbon, R. Weigel, A. Koelpin, "Promise of a Better Position", IEEE Microwave Magazine, vol.13, no.7, pp. 41-49, Nov.-Dec. 2012.

[22] F. Barbon, G. Vinci, S. Lindner, R. Weigel, A. Koelpin, "A six-port interferometer based micrometer-accuracy displacement and vibration measurement radar", Microwave Symposium Digest (MTT), 2012 IEEE MTT-S International, Conference CD, pp.1-3, June 17-22, 2012.

[23] G. Vinci, S. Lindner, F. Barbon, S. Mann, M. Hofmann, A. Duda, R. Weigel, A. Koelpin, ”SixPort Radar Sensor for Remote Respiration Rate and Heartbeat Vital-Sign Monitoring”, IEEE Transactions on Microwave Theory and Techniques, vol.61, no.5, pp.2093-2100, May 2013. 
[24] G. Vinci, S. Lindner, F. Barbon, S. Mann, R. Weigel, A. Koelpin, "Six-Port Microwave Interferometer Radar for Mechanical Vibration Analysis“, Proceedings of the European Microwave Conference, pp. 6-11, October 2013.

[25] G. Vinci, A. Koelpin, F. Barbon, R. Weigel, "Six-Port-Based Direction-Of-Arrival Detection System”, Proceedings Asia-Pacific Microwave Conference APMC 2010, pp. 1817-1820, 2010.

[26] G. Vinci, F. Barbon, R. Weigel, A. Koelpin, “A Novel, Wide Angle, High Resolution Direction-Of-Arrival Detector", Proceedings of the 8th European Radar Conference 2011, pp. 265-268, 2011.

[27]C. de la Morena-Álvarez Palencia, M. Burgos-García, "Four-octave six-port receiver and its calibration for broadband communications and software defined radios," Progress in Electromagnetics Research, vol. 116, pp. 1-21, 2011.

[28] A. Hasan, M. Helaoui, "Novel Modeling and Calibration Approach for Multi-Port Receivers Mitigating System Imperfections and Hardware Impairments," The IEEE Transactions on Microwave Theory and Techniques, vol. 60, no. 8, pp. 2644-2653, August 2012

[29] M. Helaoui, S. Boumaiza, A. Ghazel, F. M. Ghannouchi, "Power and efficiency enhancement of 3G multi-carrier amplifiers using digital signal processing with experimental validation," IEEE Transactions on Microvave Theory and Techniques, vol. 54, no. 4, pp. 1396-1404, April 2006.

[30] C. A. Hoer, K. C. Roe, C. M. Allred, "Measuring and minimizing diode detector nonlinearity," IEEE Transactions on Instrumentation and Measurements, vol. IM-25, no. 4, pp. 324-329, Dec. 1976.

[31] A. Hasan, M. Helaoui, F. M. Ghannouchi, "Dynamic Linearization of Diodes for High Speed and Peak Power Detection Applications," in 2012 IEEE MTT-S International Microwave Symposium Digest (IMS'2012), Montreal, QC, pp. 1-3, June 17-22, 2012. 
[32] H. Gomes, A. R. Testera, N. B. Carvalho, M. Fernández-Barciela, K. A. Remley, "Diode power probe measurements of wireless signals," IEEE Transactions on Microwave Theory and Techniques, vol.59, no.4, pp. 987-997, April 2011. 